\title{
Avaliação de métodos de quantificação da deformação finita por meio de simulações computacionais de deformação progressiva
}

\author{
Sérgio Wilians de Oliveira Rodrigues ${ }^{1}$ \& Frederico Meira Faleiros ${ }^{2}$
}

\begin{abstract}
Resumo Simulações computacionais de deformação progressiva por cisalhamento puro e simples aplicadas em imagens elaboradas (elipses, trama irregular e trama granoblástica) permitiram avaliar a real potencialidade dos métodos do tensor de inércia, interceptos e $R / \varphi$ (técnicas de Shimamoto \& Ikeda 1976 e Peach \& Lisle 1979) para a quantificação da deformação finita. Os resultados mostram que, em geral, os métodos avaliados apresentam-se eficientes em determinar as razões de forma e a sua orientação preferencial, com exceção de alguns casos e condições específicas, tais como taxas de deformações altas. O método do tensor de inércia, originalmente desenvolvido para análise de tramas magmáticas, apresenta resultados analíticos muito similares aos do método $R_{f} \varphi \varphi$, classicamente aplicado à analise de rochas deformadas. Ambos os métodos apresentaram boas correlações entre os valores teóricos e observados de deformação. O método dos interceptos, também idealizado para tramas magmáticas, sistematicamente fornece razões de deformação subestimadas em ensaios de cisalhamento puro, e superestimadas nos ensaios de cisalhamento simples. Contudo, apresenta bons resultados em termos de orientação. De modo geral, os testes realizados qualificam o uso dos métodos avaliados para análises de deformação.
\end{abstract}

Palavras-chave Análise da deformação, orientação preferencial de forma (OPF), tensor de inércia, método dos interceptos, $R_{f} \varphi$

\begin{abstract}
Evaluation of finite strain quantification methods by computational simulations of progressive deformation. Computational simulations of pure and simple shear progressive deformations applied on elaborated images (ellipses, irregular fabric and granoblastic fabric) permitted to evaluate the real potentiality of the methods inertia tensor, intercepts and $R_{f} / \varphi$ (technics by Shimamoto \& Ikeda 1976 and Peach \& Lisle 1979 ) in the quantification of finite strain. The results show that, in general, the evaluated methods are efficient to determine aspect ratio and preferential orientation, with exception of some cases and specific conditions, like high strain ratio. The inertia tensor method, originally developed to magmatic fabric analysis, shows analytical results very similar to those yielded by the $R / \varphi$ method, classically applied to deformed rock analysis. Both methods displayed good correlation between theoretical and observed values of deformation. The intercept method, also proposed to magmatic fabric analysis, systematically yields subestimated strain ratio on simple shear simulations and superestimated strain ratio on pure shear simulations. Nevertheless, this method displays good results in terms of orientation. In general, the tests qualify the use of the evaluated methods for strain analysis.
\end{abstract}

Keywords: strain analysis, shape preferred orientation (SPO), Inertia tensor, intercepts, $R_{f} / \varphi$

INTRODUÇÃO A utilização de métodos quantitativos propiciou grande avanço em praticamente todos os ramos da ciência, uma vez que exige considerações mais exatas, indica pontos científicos que ainda necessitam esclarecimentos e, algumas vezes, permite o reconhecimento de informações residuais menores que escapariam da atenção, mas que apontam importantes fatos (Sorby 1908).

Em geologia estrutural não foi diferente. A análise da geometria de rochas naturalmente deformadas passou por uma grande revolução desde as últimas duas décadas, em função da compreensão de que a deformação das rochas segue leis físicas e químicas bem definidas, e da utilização de métodos matemáticos e aplicação de conceitos de mecânica do contínuo (Ramsay \& Huber 1983).
A quantificação da deformação finita em rochas metamórficas constitui uma importante ferramenta em geologia estrutural, uma vez que permite caracterizar a forma, orientação e distribuição geográfica dos elipsóides de deformação de uma zona orogênica. De particular interesse nesse âmbito cita-se a possibilidade de confrontar os dados obtidos em zonas naturalmente deformadas com as previsões propostas pelos modelos teóricos de zonas de cisalhamento e transpressão/transtração (e.g. Ramsay \& Graham 1970, Ramsay 1980, Sanderson \& Marchini 1984, Tikoff \& Teyssier 1994, Robin \& Cruden 1994).

Nos últimos anos a aplicação de métodos quantitativos tem sido também utilizada para a caracterização de tramas minerais de rochas ígneas (principalmente corpos graníticos), fornecendo informações impor- 
tantes acerca de suas colocações.

Os métodos quantitativos de caracterização da trama mineral tomaram um novo impulso desde o fim da década de 90, com o desenvolvimento de programas computacionais de livre acesso, tais como Rf/ Phi (Mulchrone \& Lisle, 1999 e Mulchrone \& Meere 2001), RFPSHIM (Brandon1996), Intercepts (Launeau \& Robin2003a) e SPO (Launeau e Robin 2003b). Com a utilização desses programas a obtenção da elipse de deformação uma trama mineral tornou-se uma operação relativamente fácil e rápida.

No entanto, apesar dessa praticidade, não há um consenso ou uma avaliação que determine a melhor forma ou rotina para a utilização destes programas. Também é de fundamental interesse avaliar se os métodos de quantificação desenvolvidos para análise de tramas de rochas ígneas fornecem bons resultados em termos de razões de deformação.

Deste modo, realizamos uma série de simulações computacionais de deformação progressiva, visando avaliar os programas de cálculo de forma e orientação de elipses em análises bidimensionais (2D).

Os testes teóricos elaborados foram realizados em imagens com formas e tramas distintas nos quais foram aplicadas deformações conhecidas. Deste modo, são comparados os resultados esperados e os obtidos por meio de diferentes métodos.

MÉTODOS Razões de forma de tramas bidimensionais idealizadas (imagens digitalizadas) foram obtidas por meio da aplicação do método clássico de análise de orientação preferencial de forma: $R / \varphi$ (Ramsay1967, Ramsay \& Huber1983 e 1987) com a utilização das técnicas de Shimamoto \& Ikeda (1976) e Peach \& Lisle (1979). Também foram aplicados os métodos do tensor de inércia (Launeau \& Cruden 1998) e dos Interceptos (Launeau et al. 1990, Launeau \& Robin 1996).

Para os cálculos dos parâmetros de orientação e forma foram utilizados os programas Rf/Phi versão 1.0 (Mulchrone \& Lisle 1999 e Mulchrone \& Meere 2001), baseado na técnica de Peach \& Lisle (1979), RFPSHIM (Brandon 1996) fundamentado em Shimamoto \& Ikeda (1976), Intercepts v. 2003 (Launeau \& Robin 2003a) e SPO v. 2003 (Launeau e Robin 2003b).

Método $R / \varphi \quad$ O método $R_{f} / \varphi$ (Ramsay 1967, Ramsay $\&$ Huber 1983) pressupõe que, quando uma elipse inicial (com elipsidade $R_{i}$ ) é deformada homogeneamente, a forma resultante é também elíptica. A elipsidade final $\left(R_{f}\right)$ depende diretamente da forma e orientação da elipse inicial e da elipse de deformação. A análise é feita por meio de um gráfico, em sistema cartesiano, relacionando a razão axial de um grande número de elipses contra a orientação de seus eixos maiores. Obtém-se um gráfico simétrico (Fig. 1B) em relação à orientação média $\left(\varphi_{1}\right)$, que define a orientação do eixo maior da elipse de deformação. Sobre a linha de simetria situamse dois pontos nos quais os eixos dos grãos e da elipse de deformação são coincidentes (Fig. 1A, elipses $1 \mathrm{e}$ 2). A razão de deformação $\left(R_{s}\right)$ pode ser calculada com as equações (1) e (2), utilizando os valores máximos e mínimos da elipsidade final $\left(R_{f}\right.$ máximo e $R_{f}$ mínimo $)$ obtidos na linha de orientação média $\left(\varphi_{1}\right)$.

$$
\mathrm{R}_{\mathrm{s}}=\left(\mathrm{R}_{\mathrm{f}} \operatorname{Máx} / \mathrm{R}_{\mathrm{f}} \min \right)^{1 / 2}
$$

quando $R_{i}>R_{s}$ (o que reflete em uma distribuição normal no gráfico de $R_{f}$ versus $\varphi$ ).

$$
\mathrm{R}_{\mathrm{s}}=\left(\mathrm{R}_{\mathrm{f}} \operatorname{Máx} / \mathrm{R}_{\mathrm{f}} \min \right)^{1 / 2}
$$

quando $R_{i}<R_{s}$ (que condiciona maior simetria do gráfico de $R_{f}$ versus $\varphi$ e uma taxa maior de deformação, sendo a distribuição em forma de gota).

O gráfico de $R_{f}$ versus $\varphi$ mostra uma característica adicional das elipses ou marcadores deformados. Ocorre uma variação sistemática na orientação dos eixos maiores dos marcadores de deformação. Essa variação angular, denominada de flutuação $\left(2 \varphi_{\text {máx }}\right)$, é uma função dos valores relativos de $R_{i}$ e de $R_{c}$. Desta forma, quando $R_{s}>R_{i}$ a flutuação é bastante pequena, diminuindo gradativamente à medida que aumenta a deformação (Fig. $1 C$ e D). Para uma orientação originalmente aleatória a flutuação inicial anterior à deformação é $180^{\circ}$, isto é, as elipses não têm uma orientação preferencial. Nos casos em que a elipse de deformação tectônica tem elipsidade menor que a elipsidade inicial, a flutuação também é $180^{\circ}$. A inflexão ocorre quando $R_{s}=R_{i} \mathrm{e}$, a partir deste ponto, com um pequeno aumento da deformação, produz-se uma forte orientação (Fig. 1E)

Os comportamentos diferenciados de deformação (curvas normais ou em gota nos gráficos de $R_{f}$ versus $\varphi$ ) foram integrados por Lisle $(1977,1985,1986)$, que apresenta uma série de gráficos nos quais diferentes curvas (curvas teóricas theta) refletem variadas condições de $R_{i}, R_{f}$ e $R_{s}$. A partir de diferentes famílias de curvas theta teóricas é possível calcular as razões de deformação adaptando-se os dados de $R_{f}$ às mesmas. As técnicas de Shimamoto \& Ikeda (1976) e Peach \& Lisle (1979) ajustam matematicamente os dados de $R_{f}$ e $\varphi$ às curvas teóricas de theta a partir de testes de simetria.

Tensor de Inércia Este método foi desenvolvido por Launeau \& Cruden (1998) originalmente para quantificação de tramas de rochas magmáticas, tendo sido tipicamente aplicado para batólitos graníticos.

A razão de forma e a orientação do eixo maior de cada grão podem ser calculadas a partir do tensor de inércia de sua forma (Launeau \& Cruden, 1998). São obtidas a partir de imagens raster das seções analisadas, sendo constituídas por pixels. Se $x_{i}$ e $y_{i}$ são as coordenadas dos pixels do grão $j$ (Fig. 2) e $A$ sua área superficial (igual ao número de pixels), então o tensor de inércia ( $\mathbf{M}_{j}$ ) de sua forma é dado pela matriz (equação 3):

$$
\mathrm{Mj}=\left|\begin{array}{ll}
m_{x x j} & m_{x y j} \\
m_{x y j} & m_{y y j}
\end{array}\right|
$$




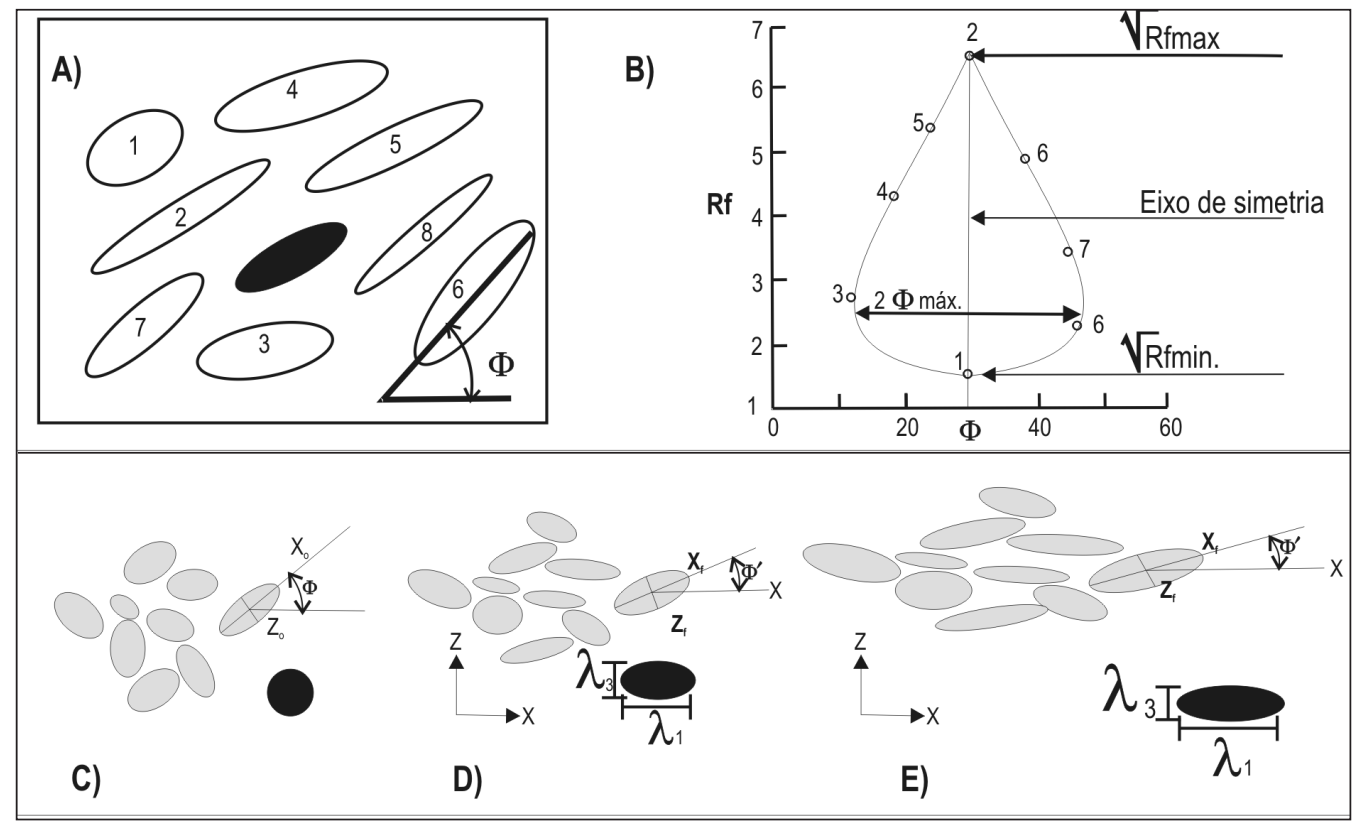

Figura 1: Deformação em marcadores elípticos. A) Deformação homogênea de elipses de razão axial originalmente constante e orientações diferentes;B) Gráfico de Rf versus $\varphi$.; a diferença entre as orientações extremas é o ângulo de flutuação( $2 \varphi)$. C) Marcadores indeformados com mesma razão inicial (Ri); D) marcadores deformados homogeneamente; E) Marcadores intensamente deformados. As elipses preenchidas representam a elipse de deformação calculada para cada exemplo apresentado. Modificado de Ramsay (1967) e Ramsay \& Huber (1983).

Onde:

$$
\begin{aligned}
& \mathrm{m}_{x x j}=1 / A \sum_{i}\left(x_{i}-x_{c}\right)^{2}, \\
& \mathrm{~m}_{x y j}=1 / A \sum_{i}\left(x_{i}-x_{c}\right)^{2}\left(y_{i}-y_{c}\right), \\
& \mathrm{m}_{y y j}=1 / A \sum_{i}\left(y_{i}-y_{c}\right)^{2}
\end{aligned}
$$

são os componentes do tensor e $x_{c}=1 / A \sum_{i} x_{i}$ e $y_{c}=1 / A$ $\sum_{i} y_{i}$ são coordenadas do centróide do grão.

A razão de forma do grão pode ser definida como $\mathrm{r}=\left(\lambda_{1} / \lambda_{2}\right)^{1 / 2}$ onde $\lambda_{1}$ e $\lambda_{2}$ são os autovalores de $\mathbf{M}_{i}$. Da mesma forma, a direção de máxima elongação do grão $(\Phi)$, é dada pelo maior autovetor de $\mathbf{M}_{j^{\prime}} \mathrm{O}$ tamanho do semi-eixo maior $(a)$, e do semi-eixo menor (b) de uma elipse representando o grão é dado por $a=$ $0,5\left(\lambda_{1}\right)^{1 / 2}$ e $b=0,5\left(\lambda_{2}\right)^{1 / 2}$ em unidade de pixels (ou em milímetros se o tamanho do pixel é conhecido).

Para correlacionar as orientações preferenciais de forma (OPF) das populações de grãos com a OPF total, introduz-se a média do tensor de inércia de $N$ formas de grãos (Launeau et al 1990),

$$
\mathrm{M}=1 / \mathrm{N} \sum_{j} M_{j}=1 / \mathrm{N}\left|\begin{array}{ll}
\sum_{j} m_{x x j} & \sum_{j} m_{x y j} \\
\sum_{j} m_{x y j} & \sum_{j} m_{y y j}
\end{array}\right|
$$

que efetivamente fornece uma OPF ponderada pela área de cada grão. A razão de forma média dos grãos $S R_{t}=\left(\lambda_{1} / \lambda_{2}\right)^{1 / 2}$ e sua orientação $\Phi$ são dadas pelos autovalores e autovetores máximos de $\mathbf{M}$, respectivamente. As dimensões médias $a_{t}$ e $b_{t}$ são calculadas da mesma forma.
A análise de população de grãos pelo tensor de inércia é bastante eficiente em delimitar as características geométricas de cada grão (eixo maior, eixo menor e orientação do seu eixo maior), principalmente em formas irregulares. Os dados individuais de cada grão podem ser utilizados em outros programas de cálculo da razão de deformação e de forma.

O programa SPO v. 2003 (Launeau \& Robin 2003 b) também calcula a $S R_{t}$ e a orientação $\Phi$ fazendo normalização pela área dos grãos de modo que cada grão apresente o mesmo peso no cálculo.

O método dos interceptos O método dos interceptos (Launeau \& Robin 1996), originalmente desenvolvido para quantificação de tramas de rochas ígneas, analisa camadas de objetos (fases) como uma população de linhas. A partir da seleção de fase a ser analisada, por exemplo um intervalo específico de tons de cinza em uma imagem, é feita uma contagem dos interceptos. A base teórica do método e suas aplicações estão detalhadas em Launeau \& Robin (1996).

A contagem dos interceptos é um método paramétrico de análise numérica da trama em uma imagem bidimensional. Ele consiste da determinação do número de interseções (número de pixels) entre o limite de um grão e sua matriz através da varredura completa da imagem por uma sucessão de linhas paralelas que giram entre 0 e $180^{\circ}$ (Fig. 3). O resultado é representado pela projeção polar da contagem de interseções (rosa de interceptos) correspondendo a cada linha de direção $\alpha$. O alongamento máximo da rosa de interceptos cor- 


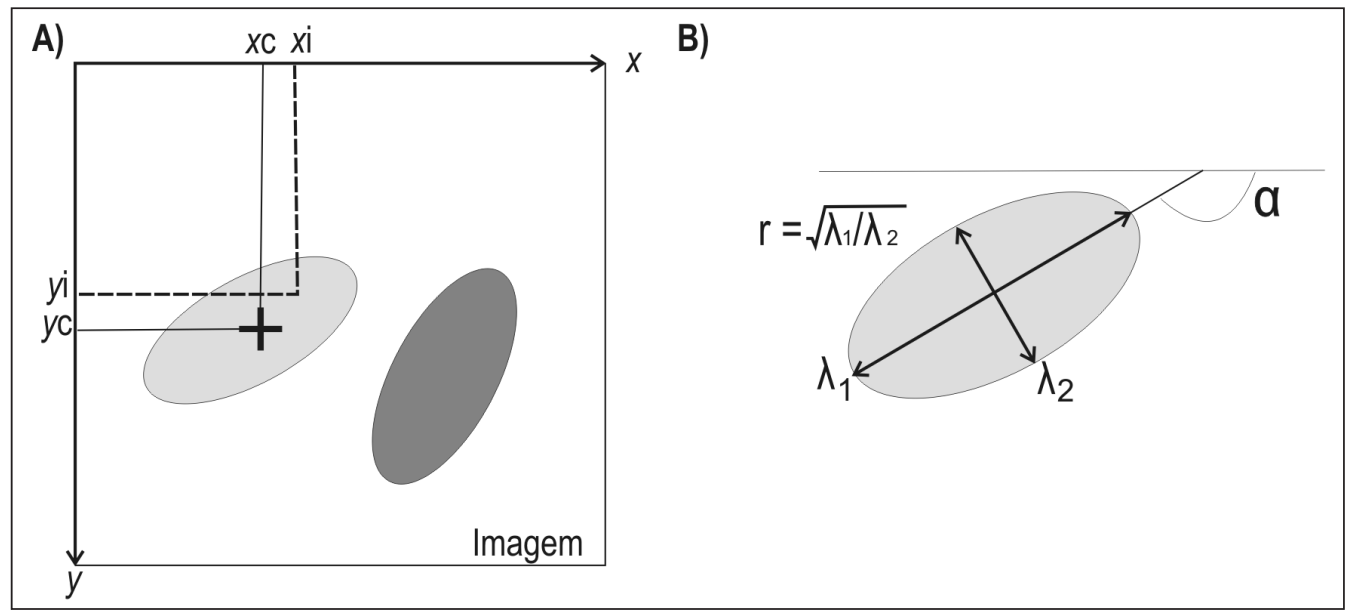

Figura 2 - Elementos do componente do tensor de inércia: A) Elementos da matriz do tensor de inércia. B) Descrição tensorial do objeto cujos autoparâmetros $\left(\lambda_{1} \mathrm{e} \lambda_{2}\right)$ definem o seixos da elipse e sua orientação $(\alpha)$ no sistema de coordenadas $x y$.

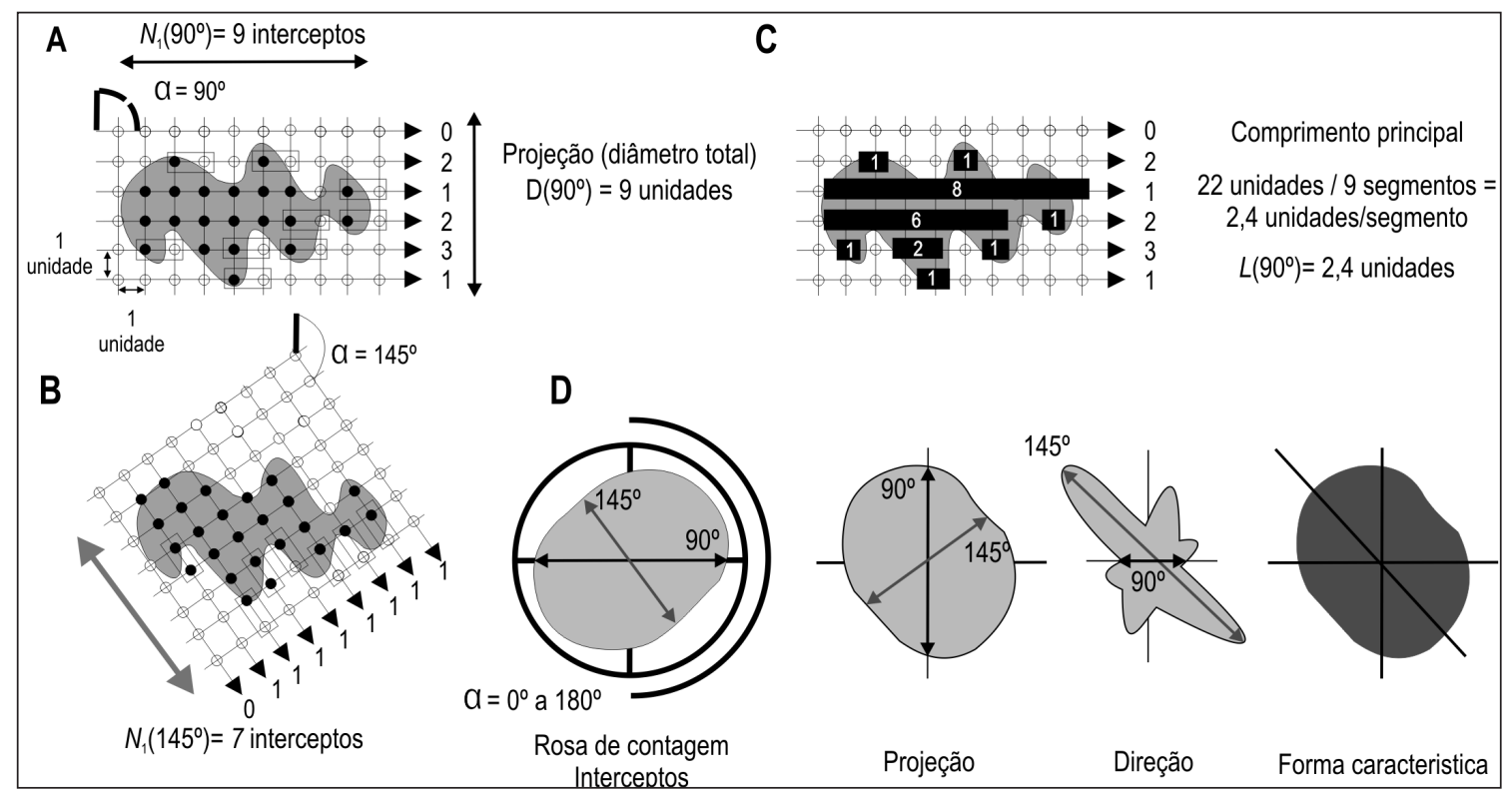

Figura 3 - Determinação da anisotropia pela contagem de interceptos (A) Malha de contagem de interceptos orientada a $90^{\circ} \mathrm{em}$ relação a um objeto (área sombreada). (B) Malha de contagem de interceptos orientada a $145^{\circ}$ em relação a um objeto. A análise de pontos é materializada por circulos vazados (fora do objeto) e preenchidos (interior do objeto). Para várias linhas regularmente espaçadas, paralelas à direção $\alpha$, o número de interceptos é $N_{l}(\alpha)$. (C) Malha de contagem de interceptos na direção $90^{\circ}$ e definição do comprimento principal (valor direcional). (D) Diagramas em rosáceas de contagem, com projeção do diâmetro total, direção e forma característica. Modificado de Launeau \& Robin (1996).

responde à direção de menor contagem. A magnitude e a orientação da anisotropia são fornecidas pela rosa de interceptos ponderada pela área total do grão. A rosa de direções, derivada da rosa de interceptos, permite ainda visualizar a geometria interna da trama através das diferentes orientações que constituem anisotropia total.

A direção principal de alongamento da população de grãos pode ser calculada utilizando os eixos de simetria fornecidos pelos autovetores da matriz $G$ dos cosenos diretores dos interceptos (Harvey \& Lax- ton 1980):

$$
G=\left|\begin{array}{cc}
\sum n \mathrm{a}_{\cos \mathrm{a}^{2}} & \sum n \mathrm{a} \cos \mathrm{a} \sin \mathrm{a} \\
\sum n \mathrm{a} \cos \mathrm{a} \sin \mathrm{a} & \sum n \mathrm{a} \sin \mathrm{a}^{2}
\end{array}\right|
$$

onde $n$ é o número total de interceptos e $n \alpha$ o número de interceptos na direção $\alpha$.

A magnitude da orientação preferencial de for- 
ma $\left(s r_{\mathrm{i}}\right)$ é fornecida pela razão entre os comprimentos médios dos interceptos $(L)$ de uma dada população computado nas direções $\alpha$ e $\alpha+\pi / 2$ onde:

$$
s r_{\mathrm{i}=} L \alpha / L \alpha+\pi / 2 \text { equação }
$$

Para uma população de grãos circulares (isotrópicos), ou quando os grãos são anisotrópicos porém distribuídos aleatoriamente, a rosa de interceptos ponderada possui um $s r_{\mathrm{i}}=1$; se a população de grãos anisotrópicos for perfeitamente alinhada, teríamos $s r_{\mathrm{i}}=r$, ou seja, a razão axial $(a / c)$ média da população de grãos.

$\mathrm{O}$ método dos interceptos destaca-se dos demais por permitir trabalhar com imagens digitais sem um tratamento digital minucioso, como o desenho dos grãos, o qual na maioria dos métodos e programas é necessário. No processamento de imagens, geralmente grãos isolados, perdem-se suas características sendo reconhecidos com um único grão. $\mathrm{O}$ método dos interceptos, por trabalhar sem a necessidade de individualizar grãos, consegue reconhecer a anisotropia original dos grãos.

AVALIAÇÃo DE MÉTOdOS DE CÁLCULO DE RAZÕES DE DEFORMAÇÃO EM TRAMAS MINERAIS Para a avaliação dos programas de cálculo de forma e orientação das elipses (2D) desenvolvemos uma série de simulações computacionais de deformação progressiva. O parâmetro utilizado para a comparação dos resultados foi a razão de forma.

Para tanto criamos uma série de imagens buscando representar diferentes tramas de rochas natu-

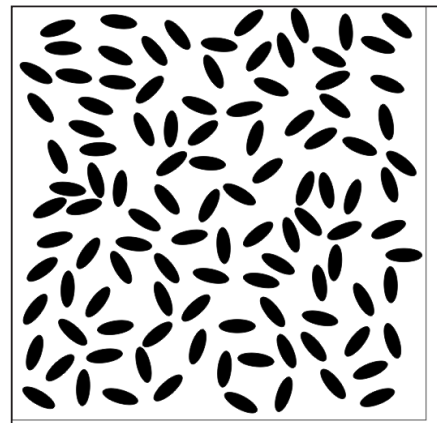

Caso 1: Elipses 1

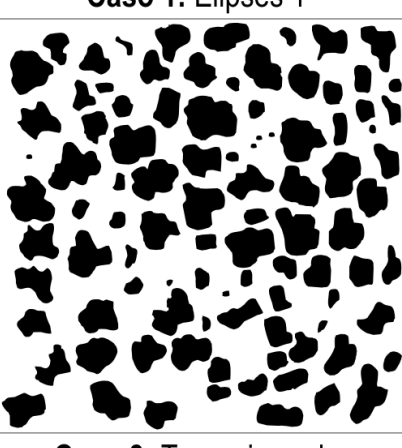

Caso 3: Trama irregular

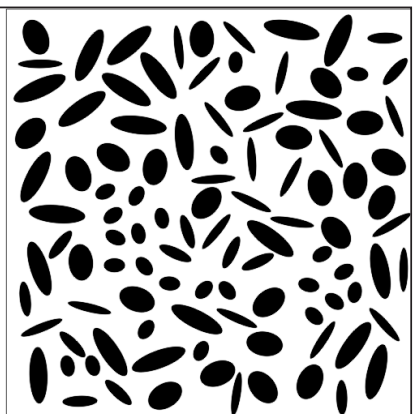

Caso 2: Elipses 2

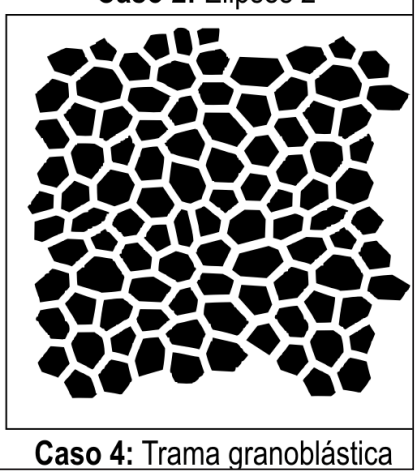

Figura 4 - Análise de 4 casos: 1 e 2, com objetos na forma de elipses; caso 3, com trama irregular e 4, com trama granoblástica.

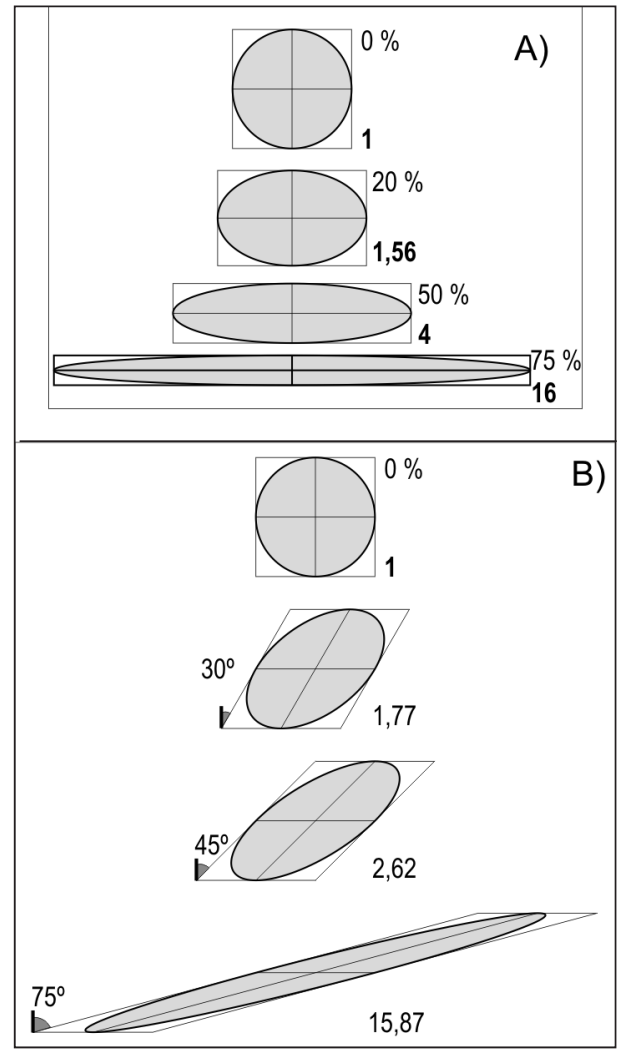

Figura 5 - Deformações impostas (A) Cisalhamento puro com as magnitudes de achatamento e razões de deformação esperadas, (B) Cisalhamento simples com o ângulo de cisalhamento (angular shear) e razões de deformação esperadas.

ralmente deformadas (Fig. 4). No computador essas imagens foram deformadas continuamente por cisalhamento puro e simples em intervalos com razões de deformação conhecidas (Fig. 5).

As imagens elaboradas (casos analisados) tentam respeitar alguns pressupostos como razão inicial de deformação próximo a 1 (ausência de orientação preferencial inicial) e número aproximado de 100 grãos em cada imagem. A razão inicial próxima a 1 justifica-se pelo fato de as razões esperadas (razões finais) serem significamente maiores quando há um componente inicial na deformação $\left(R_{r}>1\right)$.

As imagens elaboradas visaram refletir casos teóricos e reais. Os casos 1 e 2 foram escolhidos por se aproximar dos aspectos teóricos no qual o método $R / \varphi$ foi idealizado (análise da deformação de marcadores elípticos). O caso 1 (elipses 1) caracteriza-se por elipses com direções distribuídas em várias direções e com razões iniciais iguais. $\mathrm{O}$ caso 2 (elipses 2 ) também apresenta elipses com direções distribuídas em várias direções, mas com razões iniciais diferentes (três tipos de elipses). Os casos 3 e 4 espelham casos geologicamente plausíveis (trama irregular e trama granoblástica).

A figura 6 apresenta os gráficos $R / \varphi$ gerados 


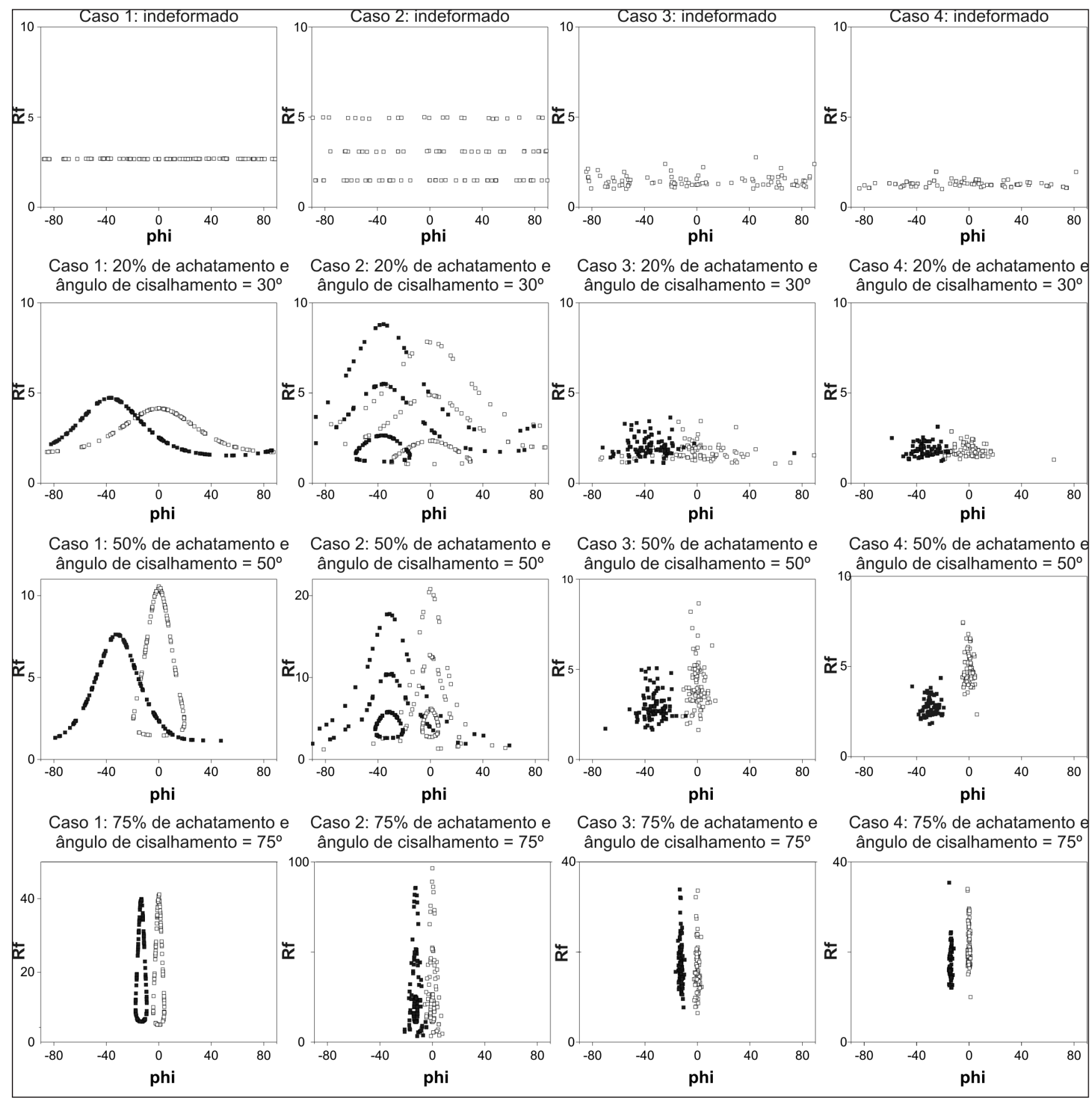

Figura 6 - Gráficos $R / \varphi$ para os casos analisados por deformação por cisalhamento puro (quadrados brancos) e simples (quadrados pretos).

para os casos analisados na deformação por cisalhamento puro.

Os gráficos $R / \varphi$ no caso 1 (elipses aleatórias) refletem o comportamento teórico esperado para a deformação, sendo observado nos estágios iniciais uma distribuição em uma curva normal e com a progressão da deformação a curva evolui para a forma de uma gota.

O caso 2 apresenta a evolução de três curvas distintas que refletem as três populações de elipses com razões axiais iniciais distintas. Cada população de elipse neste caso pode ser analisada independentemente e com maior dificuldade conjuntamente. Destaca-se nos gráficos a população de elipses mais achatadas que apresentam valores de $R_{f}$ (razões finais) superiores que as demais (algumas no limite de 100, no achatamento de $75 \%$ ). Essa população influencia significativamente no cálculo da razão de deformação $\left(R_{s}\right)$, quando utilizadas as fórmulas propostas por Ramsay (1967) (ver equações 1 e 2). Nesse caso a taxa de deformação calculada refletiria somente essa população uma vez que seus valores $R_{f}$ máximos e mínimos superporiam àqueles das demais populações de grãos.

Ambos os casos, 1 e 2, apresentam suas curvas parecidas com as curvas teóricas theta (Lisle 1977,1985 e 1986), de forma que esperaríamos uma boa correlação 
Tabela 1 - Resultados obtidos para cisalhamento simples.

\begin{tabular}{l|c|c|c|c|c|c}
\hline & \multicolumn{3}{|c|}{ Tensor de inércia } & \multicolumn{3}{c}{ Interceptos } \\
\hline Caso 1 (elipses 1) & ângulo & Razão & Erro (\%) & ângulo & Razão & Erro (\%) \\
\hline indeformado & 112,508 & 1,088 & $-\mathrm{x}-$ & 112,674 & 1,100 & $-\mathrm{x}-$ \\
\hline ângulo de cisalhamento $=30^{\circ}$ & 55,290 & 1,638 & $-7,47$ & 55,755 & 1,617 & $-8,618$ \\
\hline ângulo de cisalhamento $=50^{\circ}$ & 57,868 & 2,652 & 1,24 & 59,100 & 2,367 & $-9,673$ \\
\hline ângulo de cisalhamento $=75^{\circ}$ & 75,811 & 14,068 & $-11,35$ & 75,663 & 11,361 & $-28,409$ \\
\hline Caso 2 (elipses 2) & & & & & & \\
\hline indeformado & 171,179 & 1,020 & $-\mathrm{x}-$ & 170,048 & 1,021 & $-\mathrm{x}-$ \\
\hline ângulo de cisalhamento $=30^{\circ}$ & 52,185 & 1,776 & 0,337 & 52,145 & 1,766 & $-0,205$ \\
\hline ângulo de cisalhamento $=50^{\circ}$ & 57,868 & 2,652 & 1,235 & 57,851 & 2,619 & $-0,044$ \\
\hline ângulo de cisalhamento $=75^{\circ}$ & 75,869 & 16,754 & 5,570 & 75,730 & 12,371 & $-22,048$ \\
\hline Caso 3 (trama irregular) & & & & & & \\
\hline indeformado & 12,706 & 1,039 & $-\mathrm{x}-$ & 165,388 & 1,042 & $-\mathrm{x}-$ \\
\hline ângulo de cisalhamento $=30^{\circ}$ & 51,667 & 1,807 & 2,077 & 51,414 & 1,744 & $-1,462$ \\
\hline ângulo de cisalhamento $=50^{\circ}$ & 57,627 & 2,678 & 2,221 & 57,439 & 2,578 & $-1,607$ \\
\hline ângulo de cisalhamento $=75^{\circ}$ & 75,908 & 15,818 & $-0,327$ & 75,733 & 11,617 & $-26,799$ \\
\hline Caso 4 (trama granoblástica) & & & & & & \\
\hline indeformado & 86,317 & 1,092 & $-\mathrm{x}-$ & 87,984 & 1,093 & $-\mathrm{x}-$ \\
\hline ângulo de cisalhamento $=30^{\circ}$ & 57,138 & 1,793 & 1,292 & 57,066 & 1,773 & 0,173 \\
\hline ângulo de cisalhamento $=50^{\circ}$ & 60,422 & 2,648 & 1,058 & 60,551 & 2,604 & $-0,596$ \\
\hline ângulo de cisalhamento $=75^{\circ}$ & 76,071 & 17,021 & 7,050 & 76,099 & 12,249 & 7,87 \\
\hline
\end{tabular}

\begin{tabular}{|c|c|c|c|c|c|c|}
\hline \multirow[b]{2}{*}{ Caso 1 (elipses 1) } & \multicolumn{3}{|c|}{ Rf/Phi (Shimamoto \& Ikeda 1976) } & \multicolumn{3}{|c|}{ Rf/Phi (Peach \& Lisle 1979) } \\
\hline & ângulo & Razão & Erro $(\%)$ & ângulo & Razão & Erro $(\%)$ \\
\hline indeformado & 112,5 & 1,088 & $-\mathrm{x}-$ & 112,485 & 1,250 & $-\mathrm{x}-$ \\
\hline ângulo de cisalhamento $=30^{\circ}$ & 55,3 & 1,638 & $-7,458$ & 58,488 & 2,644 & 49,379 \\
\hline ângulo de cisalhamento $=50^{\circ}$ & 59,0 & 2,396 & $-8,550$ & 62,237 & 2,809 & 7,214 \\
\hline ângulo de cisalhamento $=75^{\circ}$ & 75,8 & 14,068 & $-11,355$ & 75,786 & 13,571 & $-14,486$ \\
\hline \multicolumn{7}{|l|}{ Caso 2 (elipses 2) } \\
\hline indeformado & 171,2 & 1,020 & $-x-$ & 76,508 & 1,100 & $-\mathrm{x}-$ \\
\hline ângulo de cisalhamento $=30^{\circ}$ & 52,2 & 1,776 & 0,339 & 51,909 & 2,532 & 43,051 \\
\hline ângulo de cisalhamento $=50^{\circ}$ & 57,9 & 2,652 & 1,221 & 57,951 & 3,228 & 23,206 \\
\hline ângulo de cisalhamento $=75^{\circ}$ & 75,9 & 16,754 & 5,570 & 75,894 & 16,500 & 3,970 \\
\hline \multicolumn{7}{|l|}{ Caso 3 (trama irregular) } \\
\hline indeformado & 16,3 & 1,038 & $-x-$ & 20,979 & 1,100 & $-\mathrm{x}-$ \\
\hline ângulo de cisalhamento $=30^{\circ}$ & 51,9 & 1,811 & 2,316 & 51,420 & 1,933 & 9,209 \\
\hline ângulo de cisalhamento $=50^{\circ}$ & 57,7 & 2,683 & 2,405 & 57,604 & 2,783 & 6,221 \\
\hline ângulo de cisalhamento $=75^{\circ}$ & 75,9 & 15,793 & $-0,485$ & 75,992 & 15,895 & 0,158 \\
\hline \multicolumn{7}{|l|}{ Caso 4 (trama granoblástica) } \\
\hline indeformado & 86,4 & 1,092 & $-\mathrm{x}-$ & 88,802 & 1,050 & $-\mathrm{x}-$ \\
\hline ângulo de cisalhamento $=30^{\circ}$ & 57,2 & 1,792 & 1,243 & 56,873 & 1,819 & 2,766 \\
\hline ângulo de cisalhamento $=50^{\circ}$ & 60,4 & 2,647 & 1,031 & 60,316 & 2,664 & 1,679 \\
\hline ângulo de cisalhamento $=75^{\circ}$ & 76,1 & 17,011 & 7,190 & 76,069 & 17,095 & 7,719 \\
\hline
\end{tabular}

entre os resultados obtidos com os programas baseados no método $R / \varphi$ (Rf/Phi v. 1 e RFPSHIM).
Os casos 3 e 4 apresentam um grande espalhamento de dados nos gráficos $R / \varphi$, mas com um com- 
Tabela 2 - Resultados obtidos para cisalhamento puro.

\begin{tabular}{|c|c|c|c|c|c|c|}
\hline & \multicolumn{3}{|c|}{ Tensor de inércia } & \multicolumn{3}{|c|}{ Interceptos } \\
\hline & ângulo & Razão & Erro $(\%)$ & ângulo & Razão & Erro $(\%)$ \\
\hline \multicolumn{7}{|l|}{ Caso 1 (elipses 1) } \\
\hline indeformado & 112,508 & 1,088 & $-x-$ & 112,674 & 1,100 & $-\mathrm{x}-$ \\
\hline $20 \%$ de achatamento & 93,258 & 1,654 & 6,030 & 93,592 & 1,657 & 6,228 \\
\hline $50 \%$ de achatamento & 90,870 & 4,174 & 4,348 & 91,061 & 4,106 & 2,662 \\
\hline $75 \%$ de achatamento & 90,206 & 16,358 & 2,235 & 90,159 & 17,734 & 10,839 \\
\hline \multicolumn{7}{|l|}{ Caso 2 (elipses 2) } \\
\hline indeformado & 171,179 & 1,020 & $-x-$ & 170,048 & 1,021 & $-\mathrm{x}-$ \\
\hline $20 \%$ de achatamento & 90,379 & 1,543 & $-1,058$ & 90,358 & 1,538 & $-1,427$ \\
\hline $50 \%$ de achatamento & 90,091 & 4,002 & 0,050 & 90,018 & 3,939 & $-1,531$ \\
\hline $75 \%$ de achatamento & 90,022 & 16,446 & 2,788 & 90,022 & 17,931 & 12,067 \\
\hline \multicolumn{7}{|c|}{ Caso 3 (trama irregular) } \\
\hline indeformado & 12,706 & 1,039 & $-x-$ & 165,388 & 1,042 & $-x-$ \\
\hline $20 \%$ de achatamento & 88,914 & 1,500 & $-3,825$ & 91,580 & 1,469 & $-5,819$ \\
\hline $50 \%$ de achatamento & 89,739 & 3,790 & $-5,258$ & 90,482 & 3,559 & $-11,032$ \\
\hline $75 \%$ de achatamento & 89,941 & 14,743 & $-7,856$ & 90,125 & 14,332 & $-10,426$ \\
\hline \multicolumn{7}{|c|}{ Caso 4 (trama granoblástica) } \\
\hline indeformado & 86,317 & 1,092 & $-x-$ & 87,984 & 1,093 & $-\mathrm{x}-$ \\
\hline $20 \%$ de achatamento & 89,427 & 1,738 & 11,438 & 89,916 & 1,722 & 10,399 \\
\hline $50 \%$ de achatamento & 89,838 & 4,646 & 16,160 & 89,998 & 4,550 & 13,758 \\
\hline $75 \%$ de achatamento & 89,955 & 20,287 & 26,796 & 90,009 & 25,530 & 59,560 \\
\hline
\end{tabular}

\begin{tabular}{|c|c|c|c|c|c|c|}
\hline \multirow[b]{2}{*}{ Caso 1 (elipses 1 ) } & \multicolumn{3}{|c|}{ Rf/Phi (Shimamoto \& Ikeda 1976) } & \multicolumn{3}{|c|}{ Rf/Phi (Peach \& Lisle 1979) } \\
\hline & ângulo & Razão & Erro $(\%)$ & ângulo & Razão & Erro $(\%)$ \\
\hline indeformado & 112,500 & 1,088 & $-x-$ & 112,485 & 1,250 & $-\mathrm{x}-$ \\
\hline $20 \%$ de achatamento & 93,300 & 1,654 & 6,026 & 96,572 & 1,750 & 12,179 \\
\hline $50 \%$ de achatamento & 90,900 & 4,174 & 4,350 & 91,426 & 4,450 & 11,250 \\
\hline $75 \%$ de achatamento & 90,200 & 16,358 & 2,238 & 90,335 & 17,250 & 7,813 \\
\hline \multicolumn{7}{|l|}{ Caso 2 (elipses 2) } \\
\hline indeformado & 171,200 & 1,020 & $-\mathrm{x}-$ & 76,508 & 1,100 & $-x-$ \\
\hline $20 \%$ de achatamento & 90,400 & 1,543 & $-1,090$ & 90,943 & 1,550 & $-0,641$ \\
\hline $50 \%$ de achatamento & 90,100 & 4,002 & 0,050 & 90,535 & 3,950 & $-1,250$ \\
\hline $75 \%$ de achatamento & 90,000 & 16,448 & 2,800 & 90,044 & 14,750 & $-7,813$ \\
\hline \multicolumn{7}{|c|}{ Caso 3 (trama irregular) } \\
\hline indeformado & 16,300 & 1,038 & $-x-$ & 20,979 & 1,100 & $-x-$ \\
\hline $20 \%$ de achatamento & 88,700 & 1,505 & $-3,526$ & 89,066 & 1,450 & $-7,051$ \\
\hline $50 \%$ de achatamento & 89,700 & 3,800 & $-5,000$ & 89,680 & 3,700 & $-7,500$ \\
\hline $75 \%$ de achatamento & 89,900 & 14,772 & $-7,675$ & 89,933 & 13,750 & $-14,063$ \\
\hline \multicolumn{7}{|c|}{ Caso 4 (trama granoblástica) } \\
\hline indeformado & 86,400 & 1,092 & $-x-$ & 88,802 & 1,050 & $-\mathrm{x}-$ \\
\hline $20 \%$ de achatamento & 89,400 & 1,739 & 11,474 & 89,849 & 1,700 & 8,974 \\
\hline $50 \%$ de achatamento & 89,800 & 4,648 & 16,200 & 89,930 & 4,550 & 13,750 \\
\hline $75 \%$ de achatamento & 90,000 & 20,293 & 26,831 & 89,979 & 19,600 & 22,500 \\
\hline
\end{tabular}




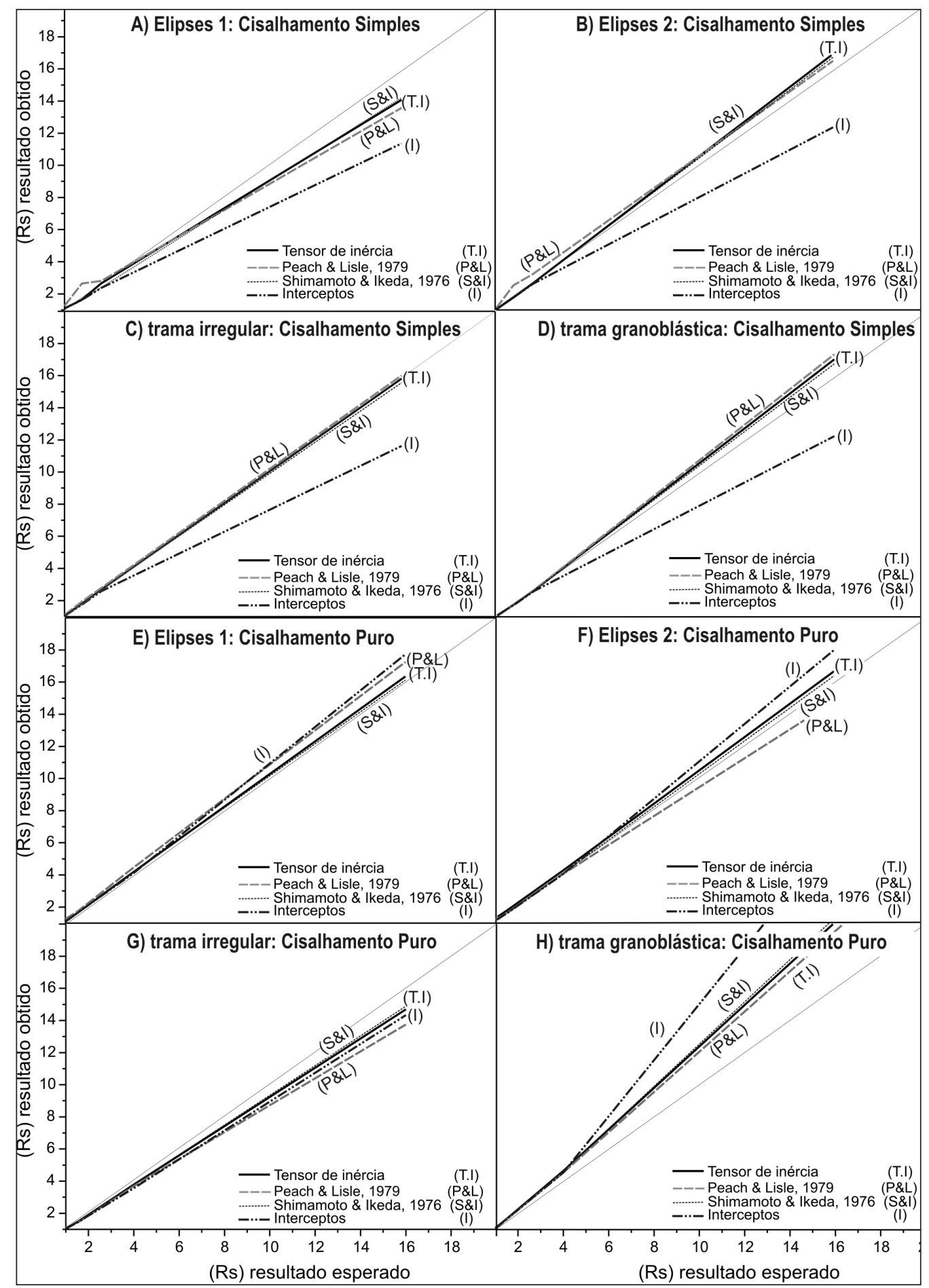

Figura 7 - Gráficos comparativos dos resultados obtidos nos ensaios de cisalhamento simples ( $A$ a $D)$ e puro $(E$ a $H)$.

portamento similar ao observado para o caso 1 . Nos estágios iniciais existe uma distribuição em uma curva normal e com a progressão da deformação a curva evolui para a forma de uma gota. No entanto, é difícil nestes casos a correlação visual com as curvas teóricas theta (Lisle 1977,1985 e 1986). Este tipo de comportamento heterogêneo das curvas $R / \varphi$ representa o padrão próximo ao esperado em casos reais.

Em ambos os tipos de cisalhamento (puro ou simples) o comportamento apresentado nos gráficos
$R / \varphi$ são similares, sendo que no cisalhamento simples há diferenciação das orientações do eixo de simetria que é similar a orientação do ângulo de cisalhamento imposto.

RESULTADOS OBTIDOS As tabelas 1 e 2 apresentam os resultados obtidos de razões forma $\left(R_{s}\right)$ e direção de cisalhamento $(\Phi)$ nos ensaios de cisalhamento puro e simples. A figura 7 apresenta os gráficos comparativos entre os resultados obtidos e esperados de $R_{s}$. A 
correlação entre os dois valores se faz por meio de retas $1: 1$. Quanto mais os resultados obtidos se aproximam da inclinação de $45^{\circ}$, melhor é a resposta do método de quantificação da razão de deformação.

$\mathrm{Na}$ deformação por cisalhamento simples (Fig. $7 A$ a $D$ ), os resultados obtidos pelos métodos do tensor de inércia e $R / \varphi$ (técnicas de Shimamoto \& Ikeda 1976 e Peach \& Lisle 1979) apresentam resultados semelhantes, bem próximos entre si, em todos os casos. Também apresentam forte correlação com os valores esperados (erro $<10 \%$ ), sendo que o caso 3 (trama irregular) é o que apresenta os resultados mais próximos aos teóricos.

$\mathrm{O}$ método dos interceptos apresentou nos casos de deformação por cisalhamento simples uma subestimação nas razões de forma $\left(R_{s}\right)$, com erros bem elevados (entre 10 e $30 \%$ ). Este método analisa as imagens considerando uma população de linhas com diferentes orientações, de forma que direções secundárias são levadas em conta no cálculo da razão de forma, podendo influenciar os valores finais. Subestimações são geradas quando há direções secundárias importantes, enquanto superestimações ocorrem quanto maior for o grau de orientação das linhas de contagens (interceptos) e menor for o peso das direções secundárias.

Os resultados dos ensaios de deformação por cisalhamento puro apresentaram comportamentos mais diversificados. Também são observados resultados semelhantes entre os métodos do tensor de inércia e $R / \varphi$ (com exceção do caso 2) e boa correlação com os valores teóricos (com exceção do Caso 4: tramas granoblástica). Em todos os métodos há uma superestimação dos resultados nos ensaios por cisalhamento puro (exceto o caso 3: trama irregular) principalmente no caso 4 (trama granoblástica). O método dos interceptos, neste caso, apresentou boas correlações entre os resultados obtidos e esperados, com exceção do caso 4, apresentando geralmente uma superestimação da deformação decorrente da maior orientação dos grãos analisados nas imagens.

De modo geral, observa-se que os métodos utilizados apresentam dificuldades no cálculo de imagens onde a magnitude de deformação é muito elevada, sendo que em alguns casos o erro entre o resultado esperado e obtido é superior a 30\% (máximo de 59,6 $\%$ no método dos interceptos, para trama granoblástica com $75 \%$ de achatamento). Para razões de deformação baixa, os métodos avaliados apresentam boas respostas com erros na faixa de $10 \%$.

Os dados de orientação preferencial de forma derivados dos métodos utilizados apresentam resultados muito próximos entre si, principalmente nas imagens de maior taxa de deformação (conseqüentemente melhor orientação preferencial).

DISCUSSÃO E CONCLUSÕES As simulações computacionais de deformação progressiva mostraram diferentes comportamentos das diferentes tramas em resposta ao cisalhamento simples e puro. Isso indica o importante papel da forma e orientação iniciais dos marcadores de deformação, bem como a orientação dos eixos de deformação na configuração final dos grãos.

De modo geral, os métodos/técnicas de cálculo da elipse seccional avaliados são eficientes para determinar as razões de forma e a sua orientação preferencial, com exceção de alguns casos e condições. Todos os métodos apresentam deficiências em registrar a deformação quando a magnitude de deformação é muito alta.

O método dos interceptos mostrou-se bastante desfavorável para o cálculo de razões de deformação para rochas submetidas ao cisalhamento simples, embora forneça bons resultados em termos de orientação. Entretanto, deve-se salientar que, em termos de deformação finita, é difícil ou impossível saber se a deformação pela qual passou uma rocha se deve ao cisalhamento simples ou puro, uma vez que ambos os mecanismos promovem deformação plana em volume constante, devendo resultar em elipsóides do tipo com k=1 (Ramsay \& Huber 1983).

O método dos interceptos apresenta características intrínsecas que o difere dos demais métodos. Por exemplo, apesar de possuir uma resposta semiquantitativa quanto aos parâmetros de forma, este método é indicado para o reconhecimento de anisotropias em análises utilizando imagens digitais (maiores detalhes em Launeau \& Robin 1996). A utilização deste método na obtenção de elipsóides de forma e deformação (análise $3 D)$ fornece resultados compatíveis com os outros métodos $(R / \varphi$ e tensor de inércia). A utilização do método dos interceptos, juntamente com os métodos do tensor de inércia e $R / \varphi$, para obtenção de elipsóides (3D) de forma e deformação atualmente é objeto de pesquisa dos autores e será abordado em um futuro trabalho.

Os resultados obtidos nos métodos do tensor de inércia e $R / \varphi$ (Shimamoto \& Ikeda 1976 e Peach $\&$ Lisle 1979) são muitos próximos entre si. Ambos os métodos apresentaram-se adequados para quantificação da deformação na maioria dos casos estudados.

Outro fator importante na utilização dos programas de cálculo da elipse seccional é a facilidade operacional de obtenção de dados. Atualmente, os programas SPO (tensor de inércia) e Intercepts (Interceptos) destacam-se por permitirem trabalhar diretamente com as imagens digitais, enquanto os programas RFPSHIM (Shimamoto \& Ikeda 1976) e Rf/Phi v. 1 (Peach \& Lisle 1979), baseados no método $R / \varphi$, trabalham com entrada de dados manuais (valores $R_{f}$ e $\varphi$ de cada grão). O programa SPO é rápido, de fácil utilização, e mostrou-se muito eficiente na quantificação da deformação, embora tenha sido idealizado para análise de tramas magmáticas. A utilização desse programa em imagens de grãos desenhados ou imagens tratadas apresentase como uma grande ferramenta para a determinação dos parâmetros de forma e orientação, podendo ainda calcular separadamente populações de grãos (por tamanho, orientação e razão de forma). 
Agradecimentos Os autores agradecem à FAPESP (processos 02/13677-4 e 02/13654-4) pelo apoio financeiro e aos professores Dr. Ginaldo Ademar da Cruz
Campanha e Dr. Carlos José Archanjo pelas discussões e incentivos durante a realização deste trabalho.

\section{Referências}

Brandon R. 1996. RFPSHIM - freeware, Yale University. Disponível em: http://earth.geology.yale.edu/ brandon/ Software/YALEDEFM/. Acessado em 30/10/2003

Harvey P.K. \& Laxton R.R. 1980. The estimation of finite strain from the orientation distribution of passively deformed linear markers: eigenvalue relationships. Tectonophysics, 70:285-307

Launeau P., Bouchez J.L., Keith B. 1990. Shape preferred orientation of object population: automatic analysis of digitized images. Tectonophysics, 180:201-211

Launeau P. \& Cruden A.R. 1998. Magmatic fabric acquisition mechanism in a syenite: results of a combined anisotropy of magnetic susceptibility and image analysis study. Journal of Geophysical Research, 103:5067-5068

Launeau P. \& Robin P.Y.F. 1996. Fabrics analysis using the intercept method. Tectonophysics, 267:91-119.

Launeau P. \& Robin P.Y.F. 2003a. INTERCEPTS v. 2003 (freeware). Université de Nantes (França)/University of Toronto (Canadá). Disponível em: http://www.sciences. univ-nantes.fr/geol/UMR6112/SPO/Intercepts.html. Acessado em 26/09/2000.

Launeau P. \& Robin P.Y.F. 2003b. SPO v. 2003 (freeware). Université de Nantes (França)/University of Toronto (Canadá). Disponível em: http://www.sciences.univnantes.fr/geol/UMR6112/SPO/SPO.html. Acessado em 13/01/2005.

Lisle R.J. 1977. Estimation of tectonic strain ratio from mean shape of deformed elliptical markers. Geologie en Mijnbouw, 56:110-144

Lisle R.J. 1985. Geological strain analysis - A manual for the Rf/phi technique. Pergamon Press, Oxford, $60 \mathrm{p}$.

Lisle R.J. 1986. The sectional strain ellipse during progressive coaxial deformation. Journal of Structural Geology, 8:809-818.

Mulchorone K.F. \& Lisle R.J. 1999. Rf/Phi v.1 (freeware). Disponível em: http://207.176.140.93/documents/oldftp/ VOL27/v27-10-12.zip. Acessado em 26/09/2000
Mulchorone K.F. \& Meere P.A. 2001. A windows program for analysis of tectonic strain using deformed elliptical markers. Computers \& Geosciences, 27:1251-1255.

Peach C.J. \& Lisle R.J. 1979. A Fortran IV program for the analysis of tectonic strain using deformed elliptical markers. Computer \& Geosciences, 5:325-334.

Ramsay J.G. 1967. Folds and fracturing of rocks. New York, McGraw Hill, 568 p.

Ramsay J.G. 1980. Shear zone geometry: a review. Journal of Structural Geology, 2:083-099.

Ramsay J.G. \& Graham R.H. 1970. Strain variation in shear belts. Canadian Journal of Earth Sciences. 7:786-813.

Ramsay J.G. \& Huber M.I. 1983. The Techniques of modern structural geology. Volume I: Strain analysis. London, Academic Press, 307 p.

Ramsay J.G. \& Huber M.I. 1987. The Techniques of Modern Structural Geology. Volume II: Folds and Fractures. London, Academic Press, 391 p.

Robin P.Y. \& Cruden A.R. 1994. Strain and vorticity patterns in ideally ductile transpression zones. Journal of Structural Geology. 16:447-466

Shimamoto I. \& Ikeda Y. 1976. A simple algebraic method for strain estimation from deformed ellipsoidal methods. Tectonophysics. 36:315-317

Sanderson D.J. \& Marchini, W.R.D. 1984. Transpression. Journal of Structural Geology. 6:449-458.

Sorby H.C. 1908. On the application of quantitative methods to the study of the structure and history of rocks: Journal of the Geological Society of London. 64:171-232.

Tikoff B. \& Teyssier C. 1994. Strain modeling of displacement-field partitioning in transpressional orogens. Journal of Structural Geology. 16:1575-1588.

Manuscrito AE-076/2006

Submetido em 26 de janeiro de 2007 Aceito em 17 de agosto de 2007 